S. A. Coronel, J. Melguizo-Gavilanes, S. Jones and J. E Shepherd.

Temperature field measurements of thermal boundary layer and wake of moving hot spheres using interferometry.

Experimental Fluid and Thermal Science, 90:76-83, 2018.

http://dx.doi.org/10.1016/j.expthermflusci.2017.08.031.

\title{
Temperature Field Measurements of Thermal Boundary Layer and Wake of Moving Hot Spheres using Interferometry
}

\author{
Stephanie A. Coronel*, Josué Melguizo-Gavilanes, Silken Jones, Joseph E. \\ Shepherd \\ Graduate Aerospace Laboratories, California Institute of Technology, Pasadena, CA 91125, USA
}

\begin{abstract}
The methodology used to post-process a raw interferogram of a hot moving sphere falling in an inert nitrogen environment is presented. The steps taken to obtain the temperature field around the hot sphere are explained in detail. These are: (i) noise removal; (ii) phase demodulation; (iii) phase unwrapping; (iv) bias removal; and (v) Abel transform. All the typical features of the flow are revealed such as growth of the thermal boundary layer, shallower temperature gradients were the flow separates, and a hot wake in the recirculation zone. For validation of the methodology, the temperature field is compared against numerical simulations and found to be in excellent qualitative and quantitative agreement all around except at the front and rear stagnation points. The difficulties encountered with resolving these regions are discussed. Overall, interferometry is found to be an excellent tool for resolving thermal flows, including thin regions, such as thermal boundary layers.
\end{abstract}

Keywords: Thermal boundary layer, Sphere, Interferometry

*Corresponding author: coronel@ caltech.edu 


\section{Introduction}

Interferometry is an optical technique for making measurements by interfering electromagnetic waves with each other. Typical measurements that can be made with interferometry are: distances, displacements and vibrations, testing of optical systems, gas flows and plasmas, microscopy, measurements of temperature, pressure, electrical and magnetic fields, rotation sensing, and high resolution spectroscopy, to name a few [1].

In combustion applications, interferometry has typically been used for making temperature measurements in steady burners $[2,3,4,5,6]$. This makes for simple post-processing of each image since time averaging can be performed to obtain the final result. However, most combustion applications are not steady, hence developing the ability to resolve transients during combustion events is important. Take for example thermal ignition. This is a process that commences with a small hot gas kernel that eventually develops into a self-propagating flame.

Getting visual and quantitative information out of experiments is not necessarily trivial, but with recent advances in visualization techniques and computing power for post-processing, spatially and temporally resolved field measurements are becoming more common. Recent efforts at the Explosion Dynamics Laboratory (EDL) of Caltech have explored interferometry as a means to extract temperature/density fields during ignition experiments using a stationary (commercial glow plug) [7, 8] and moving (sphere) hot surfaces [9] with satisfactory results.

Detailed methodologies describing the application of interferometry to unsteady combustion phenomena are still lacking in the literature. Therefore, as a first step towards filling this gap, we will discuss some of the tools developed at the EDL to make temperature field measurements for moving hot surfaces. Specif- 
ically, the main focus of this manuscript is to describe the methodology used and show the validation of temperature measurements obtained with shearing interferometry using a hot sphere of $6 \mathrm{~mm}$ in diameter falling through an inert gas (nitrogen).

\section{Technical Approach}

\subsection{Interferometry Background}

A sketch of a shearing interferometer is shown in Fig. 1. Laser light passes through a linear polarizer whose plane of polarization $(\mathrm{P})$ is oriented $45^{\circ}$ with respect to the $x-y$ plane; the polarizer produces equal magnitude electric vector components, $E_{x}$ and $E_{y}$, lying along the $x$ and $y$ axes [10]. The polarized light is incident on a lens (L) that focuses the beam onto a Wollaston prism (WP). The Wollaston prism consists of two quartz prisms (quartz has the property of birefringence; different indices of refraction along the crystallographic axes) with optical axes that are perpendicular to each other. The Wollaston prism causes the rays associated with the two perpendicular electric field components (optical polarizations) to diverge as they leave the prism; an illustration of the operation of the prism is shown in Fig. 2. If the prism is placed at the focal point of the lens, the two rays are in phase with each other but separated and orthogonally polarized to each other; if the prism is placed away from the focal point, the two rays are out of phase [11]. The separation distance, $\varepsilon$, between the two rays is given by Snell's law of refraction[11],

$$
\varepsilon=2 \alpha\left(n_{e}-n_{o}\right),
$$


where $\alpha$ (assumed to be small) is the prism angle, and $n_{e}$ and $n_{o}$ are the refractive indices of the extraordinary and ordinary rays, respectively, in the prism material. For light with a wavelength of $589 \mathrm{~nm}, n_{e}=1.5553, n_{o}=1.4864$ [11], and for $\alpha=19^{\circ}$, the separation distance $\varepsilon$ is $0.3-0.4^{\circ}$.

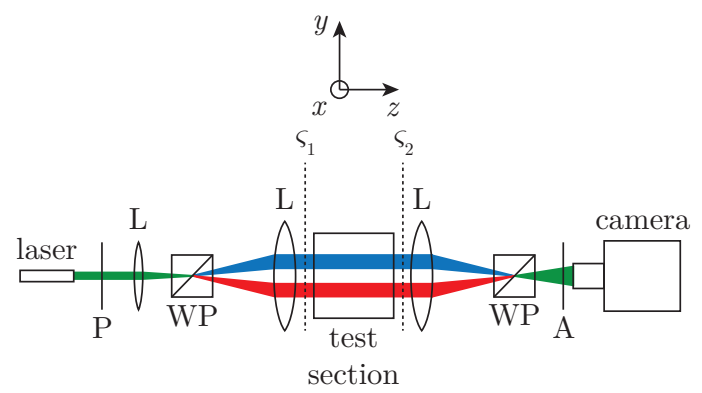

Figure 1: Schematic of shearing interferometer setup.

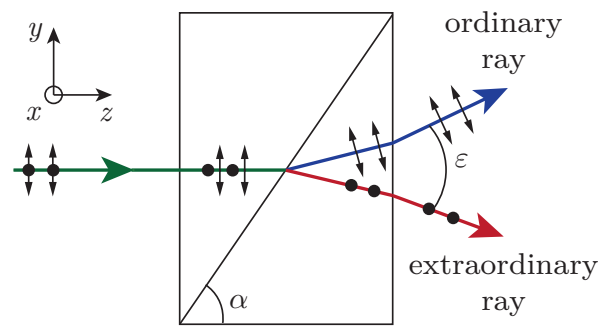

Figure 2: Illustration of Wollaston prism operation.

The two beams pass through the test section, shown in Fig. 1, and are focused onto a second Wollaston prism that is $180^{\circ}$ out of phase from the first Wollaston prism. The second prism is there to recombine the two beams; if the prism is placed at the focal point, the two beams will be in phase with each other. Finally, an interference pattern is created after the recombined beam passes through an analyzer (A). Examples of said interference patterns are shown in Fig. 5. 
The interferograms obtained with a shearing interferometer represent the optical path length difference between light traveling through a field of view with refractivity $n(z)$ (blue beam in Fig. 1) and light traveling through a reference field with refractivity $n_{0}$ (red beam in Fig. 1), see Fig. 3. The difference in phase, $\Delta \varphi$, is related to the index of refraction by,

$$
\Delta \varphi=\varphi-\varphi_{0}=\frac{2 \pi}{\lambda} \int_{\zeta_{1}}^{\zeta_{2}}\left[n(z)-n_{0}\right] \mathrm{d} z
$$

where $\zeta_{1}$ and $\zeta_{2}$ are the locations along the $z$-axis where a ray enters and leaves the test section, respectively, and $\lambda$ is the wavelength of the light in a vacuum. If the index of refraction is cylindrically symmetric, as illustrated in Fig. 3, the Abel transform, Eq. 3, can be used to relate a line of sight integrated quantity to a radially distributed one [12].

$$
F(x)=2 \int_{x}^{\infty} \frac{f(r) r}{\left(r^{2}-x^{2}\right)^{1 / 2}} \mathrm{~d} r .
$$

The inverse Abel transform is given by [12] as,

$$
f(r)=-\frac{1}{\pi} \int_{r}^{\infty} \frac{\mathrm{d} F}{\mathrm{~d} x} \frac{\mathrm{d} x}{\left(x^{2}-r^{2}\right)^{1 / 2}},
$$

where in the context of index of refraction and optical phase difference,

$$
\begin{aligned}
f(r) & =\frac{2 \pi}{\lambda}\left[n(r)-n_{0}\right] \\
F(x) & =\Delta \varphi .
\end{aligned}
$$

The density of the medium can be calculated by using the Gladstone-Dale 

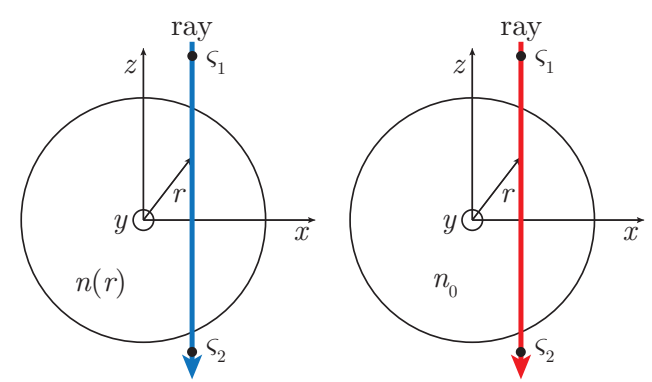

Figure 3: Rays passing through symmetric planes with index of refraction $n(r)$ (left) and $n_{0}$ (right).

relation, Eq. 6, where $K$ is the Gladstone-Dale constant specific to the gas. For nitrogen at $T=300 \mathrm{~K}$ and $P=101325 \mathrm{~Pa}, K=2.40109490653 \times 10^{-4} \mathrm{~m}^{3} / \mathrm{kg}$ [13].

$$
n-1=K \rho
$$

Finally, the temperature of the gas can be obtained through the equation of thermodynamic state:

$$
T=P W / \rho \tilde{R},
$$

where $P$ is the thermodynamic pressure, $W$ is the gas mixture molecular weight, and $\tilde{R}$ is the universal gas constant.

The technical background described above will be used to measure the gas temperature fields around a falling hot sphere. The experimental setup is explained next.

\subsection{Experimental Setup}

The experiments were performed in a closed, cylindrical, stainless steel vessel with a volume of approximately $22 \mathrm{~L}$, shown in Fig. 4 . The vessel has a height of 
$37.5 \mathrm{~cm}$ and an inner diameter of $30.2 \mathrm{~cm}$. Two parallel flanges are used to mount windows for visualization, the windows have a diameter of approximately $12 \mathrm{~cm}$. Above the vessel sits a cylindrical aluminum chamber with a volume of approximately $0.1 \mathrm{~L}$, also shown in Fig. 4. The small chamber has an inner diameter of $4 \mathrm{~cm}$ and a height of $8.9 \mathrm{~cm}$. The aluminum chamber is used to contain a sphere; it has two parallel flanges that are used to mount titanium supports, one of which can be actuated linearly through a double acting pneumatic actuator, to hold a sphere in place. The other two sides of the chamber hold Zinc-Selenide (ZnSe) windows, the field of view is approximately $1.9 \mathrm{~cm}$. Attached to the bottom of the small chamber is a hollow cylinder (inner diameter of $2.3 \mathrm{~cm}$ ) that protrudes into the inside of the stainless steel vessel; the chamber, cylinder and vessel make up a single volume.

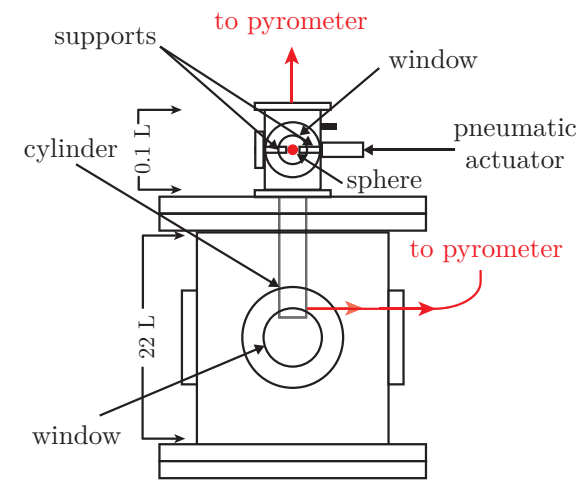

Figure 4: Illustration of experimental setup along with labeled components.

A sphere is placed in the small chamber and held by the titanium supports, a remotely controlled plumbing system is used to evacuate the combustion vessel to less than $7 \mathrm{~Pa}$ and fill it with the test mixture. A Heise manometer with a precise digital readout measures the static pressure so the gases can be filled to within 10 
$\mathrm{Pa}$ of the desired gas pressure, providing precise control over the mixture composition. Typically this experimental setup is used for ignition experiments by moving hot spheres, however, here we use it to measure the temperature of an inert gas, nitrogen, surrounding a moving hot sphere as a means of validating the methodology described in the previous subsection. The sphere surface is irradiated on opposite sides with a continuous-wave $(\mathrm{cw}) \mathrm{CO}_{2}$ laser that has a maximum power output of $80 \mathrm{~W}$ with an emission wavelength of $10.6 \mu \mathrm{m}$. During heating, a two-color pyrometer is used for making non-contact temperature measurements of the sphere surface. A Proportional-Integral-Derivative (PID) feedback controller uses the pyrometer output to adjust the laser thereby allowing precise control of the sphere surface temperature during heating. Once the desired sphere surface temperature is reached, one of the titanium supports retracts allowing the sphere to fall. The sphere travels through the cylinder and then exits into the vessel and comes into the field of view of the combustion vessel windows; a final measurement of the sphere surface temperature is made right before the sphere exits the cylinder, shown in Fig. 4. The bottom end of the cylinder is vertically aligned with the top of the vessel windows allowing for visualization of the falling sphere as it exits the cylinder.

Figure 5 (a) shows a finite fringe interferogram, where the horizontal fringes (undisturbed medium) correspond to a finite value of initial optical phase difference between the reference beam and disturbed beam. Figure 5 (b) shows an infinite fringe interferogram, where the undisturbed part of the image corresponds to a value of zero in the initial optical phase difference between the reference beam and disturbed beam. The intensity, $I$, of the two-dimensional fringe patterns observed in Fig. 5 is represented by an amplitude and frequency modulated 
function,

$$
I(x, y)=a(x, y)+b(x, y) \cos (\Delta \varphi(x, y))
$$

where $a$ represents the background illumination and noise, $b$ is the amplitude, and $\varphi$ is the phase [14]. The phase demodulation of the interferograms, i.e. obtaining $\Delta \varphi$, is accomplished by using the 2D Windowed Fourier Filtering method (WFF2) [15] described in Section 4.2. The current study will only cover the image processing procedure of the finite fringe interferograms.

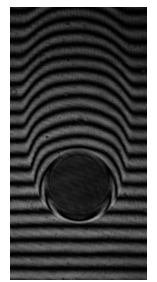

(a) Finite fringe

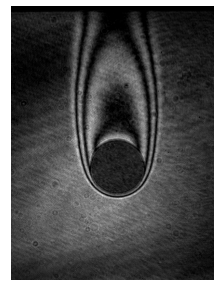

(b) Infinite fringe

Figure 5: (a) Finite fringe and (b) infinite fringe interferograms of thermal boundary layer and wake surrounding falling hot spheres.

The interferogram of Fig. 5 (a) shows a sharp shift in the fringes in the vicinity of the hot sphere; this shift corresponds to the edge of the thermal boundary layer. The fringe shift is more subtle in the wake of the sphere which corresponds to the region of flow recirculation, where temperature gradients are not as pronounced. The flow field appears axisymmetric about the path of the sphere motion; the axisymmetry is a necessary requirement to be able to use the inverse Abel transform to determine the radial distribution of the index of refraction.

Figure 6 shows a $6 \mathrm{~mm}$ diameter sphere with a surface temperature of approximately $1400 \mathrm{~K}$ falling at $2.4 \mathrm{~m} / \mathrm{s}$ in $\mathrm{N}_{2}$. A section of this sequence of images $(11.0-11.4 \mathrm{~ms})$ is analyzed in the following section. Note that this particular 
flow is not characterized by a unique Reynolds number, $\operatorname{Re}=U d / \nu$, where $U$ is the sphere velocity, $d$ is the sphere diameter, and $\nu$ is the kinematic viscosity. This is due to the strong dependence of $\nu$ on temperature, increasing by roughly one order of magnitude between $300 \mathrm{~K}$ and $1300 \mathrm{~K}$, from $2 \times 10^{-5} \mathrm{~m}^{2} / \mathrm{s}$ to $1.75 \times 10^{-4}$ $\mathrm{m}^{2} / \mathrm{s}$. This ultimately results in a Re variation between $\sim 600$ when taking the $\nu$ value at $300 \mathrm{~K}$ to less than 100 when the $\nu$ value at $1300 \mathrm{~K}$ is used. For this study, the Re number is based on the film properties which yields a value of 155 . As discussed by Johnson and Patel [16], flows are steady and axisymmetric and have a wake that is composed of a steady toroidal vortex for $20<\operatorname{Re}<210$ in uniform temperature flow.

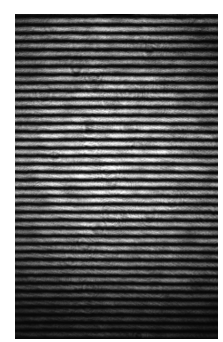

$0 \mathrm{~ms}$

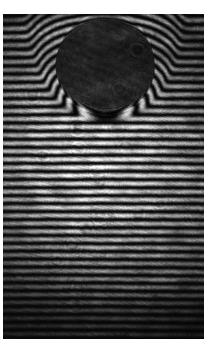

$3.8 \mathrm{~ms}$

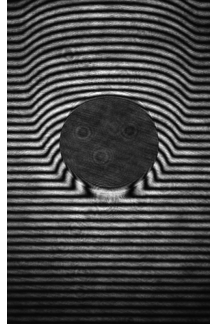

$7.1 \mathrm{~ms}$

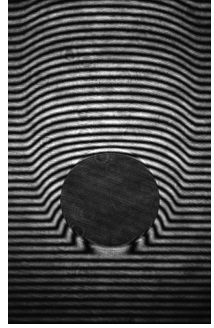

$9.1 \mathrm{~ms}$

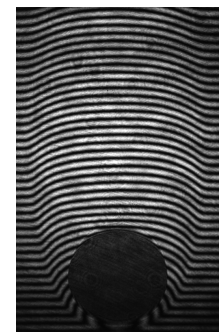

$11.5 \mathrm{~ms}$

Figure 6: Interferograms of falling $6 \mathrm{~mm}$ diameter sphere in $\mathrm{N}_{2}$; the surface temperature of the sphere is approximately $1400 \mathrm{~K}$.

\section{Post-Processing}

The sequence of steps taken to obtain an experimental temperature field from a raw interferogram is shown in Fig. 7. The steps are: (i) removal of high frequency noise using a Gaussian filter; (ii) phase demodulation; (iii) phase unwrapping; (iv) bias removal; (v) Abel transform. 


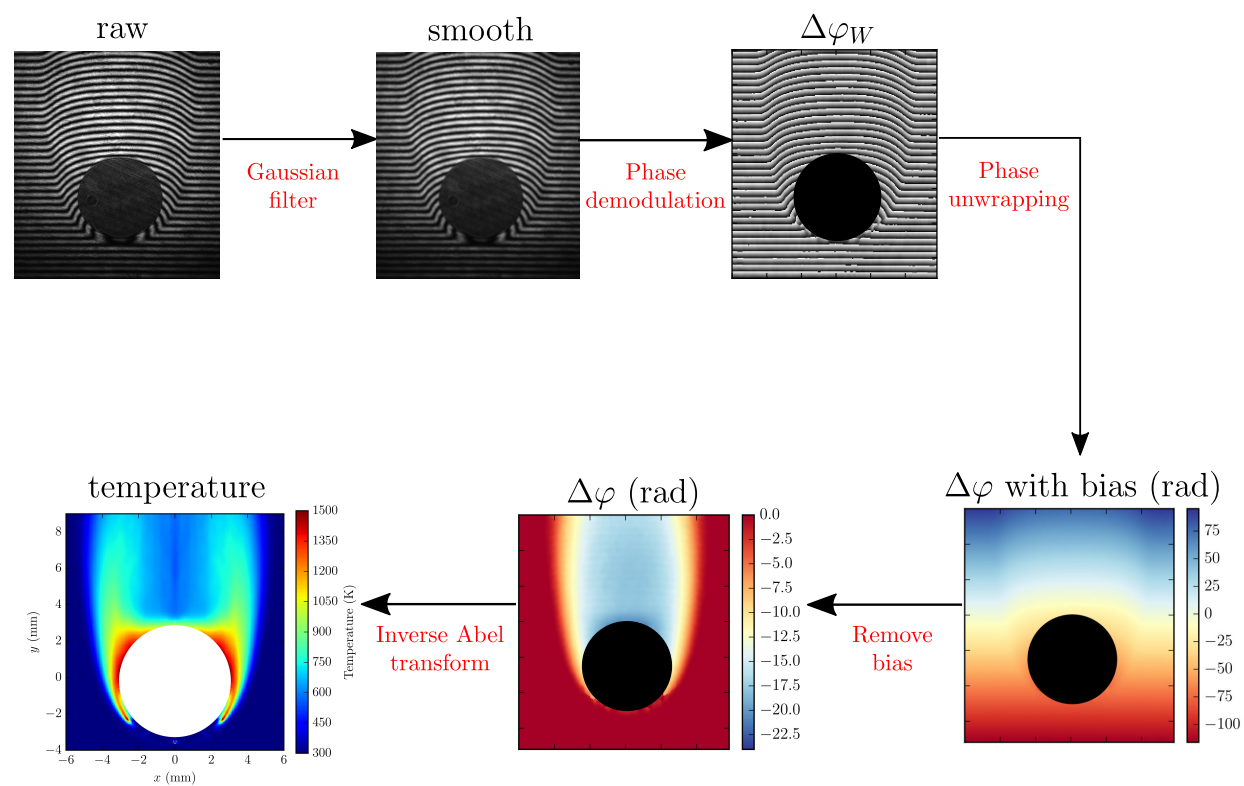

Figure 7: Flow chart of post-processing steps.

\subsection{Noise Removal}

Before performing the phase demodulation, a Gaussian filter is applied to the raw interferogram; the resulting image is labeled smooth in Fig. 7. The objective of the Gaussian filter is to remove high frequency noise present in the image. The noise removal is shown quantitatively in Fig. 8 in terms of the log of the absolute value of the Fourier spectrum for the original and Gaussian filtered images. The histogram shows the filtered high frequency signals that are associated with noise; the frequency peak shifts from $7.1 \mathrm{px}^{-1}$ (original) to $5.5 \mathrm{px}-1$ after the filter is applied.

\subsection{Phase Demodulation}

The phase demodulation, that is obtaining $\Delta \varphi$, is a accomplished by using the Windowed Fourier Filtering (WFF2) Method. Details of the method algorithm 


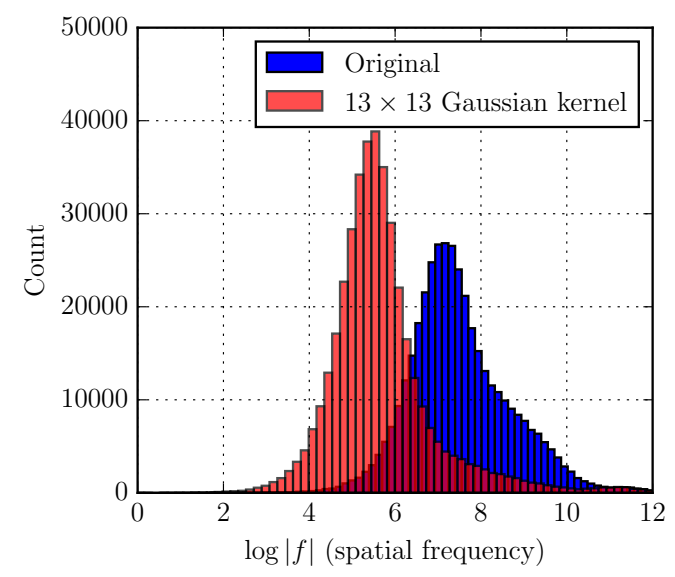

Figure 8: Histogram of $\log |f|$ taken from the raw and smooth images of Fig. 7.

are presented in [15]. This section describes the method used to extract the phase from the experimental interferogram shown in Fig. 7, labeled raw.

The phase demodulation is started by using the following windowed Fourier basis,

$$
g_{\xi_{x}, \xi_{y}}(x, y)=g(x, y) e^{i\left(\xi_{x} x+\xi_{y} y\right)}
$$

where $g(x, y)$ is a Gaussian window,

$$
g(x, y)=\frac{1}{2 \pi \sqrt{\sigma_{x} \sigma_{y}}} e^{-x^{2} / 2 \sigma_{x}^{2}-y^{2} / 2 \sigma_{y}^{2}}
$$

The 2D continuous windowed Fourier transform (WFT), $F_{W}$ and inverse WFT, $f$, can be expressed in terms of the convolution operator $\otimes$ and written as,

$$
\begin{gathered}
F_{W}\left(u, v ; \xi_{x}, \xi_{y}\right)=f(u, v) \otimes g_{\xi_{x}, \xi_{y}}(x, y) \\
f(x, y)=\frac{1}{4 \pi^{2}} \int_{-\infty}^{\infty} \int_{-\infty}^{\infty} F_{W}\left(u, v ; \xi_{x}, \xi_{y}\right) \otimes g_{\xi_{x}, \xi_{y}}(x, y) \mathrm{d} \xi_{x} \mathrm{~d} \xi_{y} .
\end{gathered}
$$


The discrete form of Eq. 12 is written as,

$$
f(x, y)=\frac{\xi_{x}^{(i)} \xi_{y}^{(i)}}{4 \pi^{2}} \sum_{\xi_{y}=-\pi}^{\pi} \sum_{\xi_{x}=-\pi}^{\pi} F_{W}\left(u, v ; \xi_{x}, \xi_{y}\right) \otimes g_{\xi_{x}, \xi_{y}}(x, y)
$$

where $\xi_{x}^{(i)}$ and $\xi_{y}^{(i)}$ are the sampling intervals of $\xi_{x}$ and $\xi_{y}$. The windowed Fourier coefficient measures the similarity between a section of the signal and the windowed Fourier kernel given in Eq. 9. The coefficients are high if the signal is similar to the windowed Fourier kernel, and small if the section of the signal consists of noise. To accurately reconstruct Eq. 13, the coefficients that correspond to the signal noise need to be eliminated. This is done by setting a predetermined threshold, thr; coefficients lower than the threshold are discarded and not used in the reconstruction. Therefore,

$$
\bar{f}(x, y)=\frac{\xi_{x}^{(i)} \xi_{y}^{(i)}}{4 \pi^{2}} \sum_{\xi_{y}=-\pi}^{\pi} \sum_{\xi_{x}=-\pi}^{\pi} \overline{F_{W}}\left(u, v ; \xi_{x}, \xi_{y}\right) \otimes g_{\xi_{x}, \xi_{y}}(x, y)
$$

where $\overline{F_{W}}\left(u, v ; \xi_{x}, \xi_{y}\right)$ denotes the thresholded spectrum,

$$
\overline{F_{W}}\left(u, v ; \xi_{x}, \xi_{y}\right)= \begin{cases}F_{W}\left(u, v ; \xi_{x}, \xi_{y}\right), & \text { if }\left|F_{W}\left(u, v ; \xi_{x}, \xi_{y}\right)\right| \geq t h r \\ 0, & \text { if }\left|F_{W}\left(u, v ; \xi_{x}, \xi_{y}\right)\right|<t h r\end{cases}
$$

The thresholded phase is found by,

$$
\overline{\Delta \varphi_{W}}(x, y)=\angle \bar{f}(x, y)
$$

where $\angle \bar{f}(x, y)$ is the angle of the thresholded signal. The subscript $W$ (wrapped) in Eq. 15 corresponds to an optical phase difference that is bounded between $-\pi$ 
and $\pi$, modulo $2 \pi$. The wrapped optical phase detected using the method above is labeled wrapped in Fig. 7 and a time sequence of images in shown in Fig. 9.

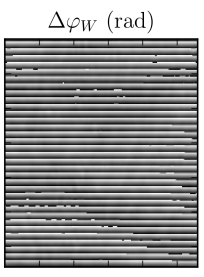

$0 \mathrm{~ms}$

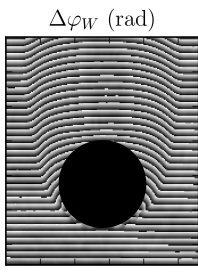

$11.0 \mathrm{~ms}$

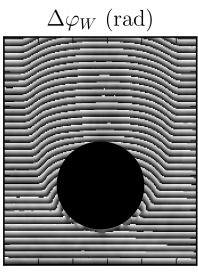

$11.1 \mathrm{~ms}$

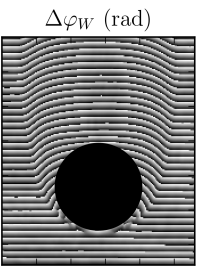

$11.2 \mathrm{~ms}$

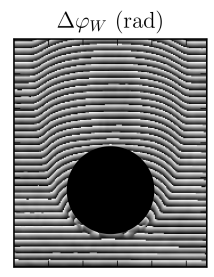

$11.3 \mathrm{~ms}$

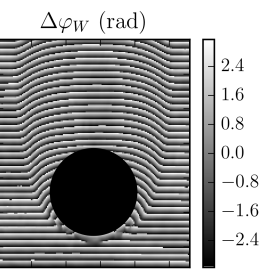

$11.4 \mathrm{~ms}$

Figure 9: Experimental wrapped optical phase difference sequence.

\subsection{Phase Unwrapping}

To construct a continuous optical phase difference, a quality guided phase map using a flood-filling algorithm was used to unwrap the phase [17]. Several methods exist for performing phase unwrapping, one of them is the path following algorithm. Within the path following method there are fixed-path and quality guided algorithms. The quality guided algorithm uses a quality map to determined the path along which the phase unwrapping is performed. The quality map, $Q(x, y)$, is given by,

$$
Q(x, y)=1-\frac{\Delta \phi_{r}(x, y)}{2 \pi}
$$

where $\phi_{r}$ is the wrapped thresholded phase $\overline{\Delta \varphi_{W}}$ and $\Delta \phi_{r}$ is the phase variance calculated from Eq. 17.

$$
\begin{aligned}
\Delta \phi_{r}(x, y)= & \frac{1}{4}\left(\left|\phi_{r}(x, y)-\phi_{r}(x, y-\Delta y)\right|+\left|\phi_{r}(x, y)-\phi_{r}(x, y+\Delta y)\right|\right. \\
& \left.+\left|\phi_{r}(x, y)-\phi_{r}(x-\Delta x, y)\right|+\left|\phi_{r}(x, y)-\phi_{r}(x+\Delta x, y)\right|\right)
\end{aligned}
$$


To begin the quality guided algorithm, a pixel with the highest quality $Q$ is chosen; the phase is unwrapped at this location. The unwrapped phase is denoted by $\phi$. Then, the quality of the 4 pixels surrounding this unwrapped location is checked, and the pixel with the highest quality is chosen next. At this chosen location the phase is once again unwrapped using Eq. 18.

$$
\phi(n)= \begin{cases}\phi(n-1)+\Delta \phi_{r}(n)+2 \pi, & \text { if } \Delta \phi_{r}(n) \leq-\pi \\ \phi(n-1)+\Delta \phi_{r}(n), & \text { if } \pi<\Delta \phi_{r}(n)<\pi \\ \phi(n-1)+\Delta \phi_{r}(n)-2 \pi, & \text { if } \Delta \phi_{r}(n) \geq-\pi\end{cases}
$$

where $n$ is the chosen pixel location and $\phi(n)$ is the unwrapped phase at that location. The unwrapped optical phase difference in the following sections is represented by $\Delta \varphi$. The unwrapped optical phase difference is labeled unwrapped in Fig. 7 and a sequence of unwrapped images are shown in Fig. 10.

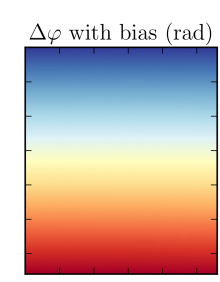

$0 \mathrm{~ms}$

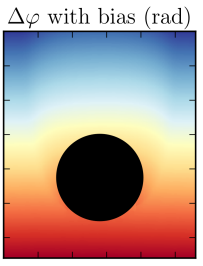

$11.0 \mathrm{~ms}$

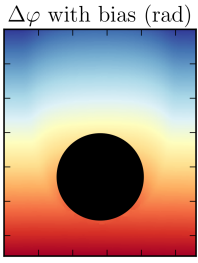

$11.1 \mathrm{~ms}$

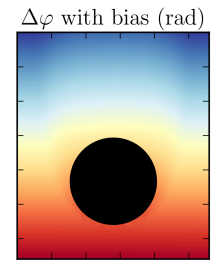

$11.2 \mathrm{~ms}$

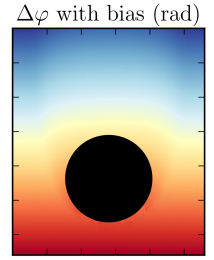

$11.3 \mathrm{~ms}$

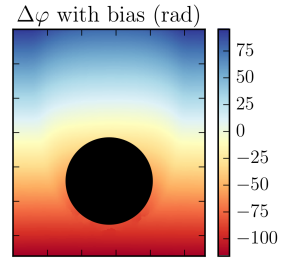

$11.4 \mathrm{~ms}$

Figure 10: Experimental unwrapped optical phase difference sequence.

\subsection{Bias Removal}

To obtain the optical phase difference, $\Delta \varphi$, the bias shown by the unwrapped optical phase difference in the undisturbed image in Fig. 10 at 0 ms needs to be removed from each subsequent image. The resulting optical phase difference is shown in Fig. 11. 


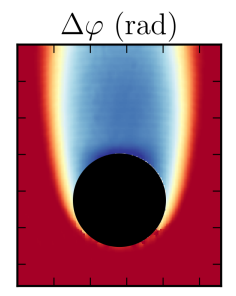

$11.0 \mathrm{~ms}$

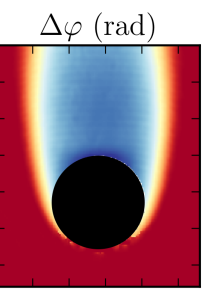

$11.1 \mathrm{~ms}$

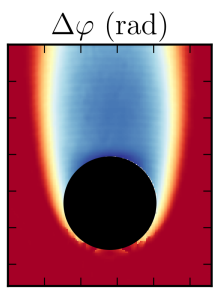

$11.2 \mathrm{~ms}$

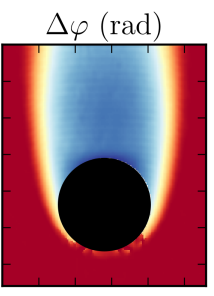

$11.3 \mathrm{~ms}$

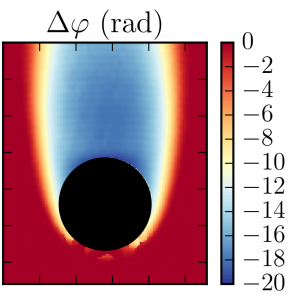

$11.4 \mathrm{~ms}$

Figure 11: Experimental optical phase difference sequence.

Before applying the inverse Abel transform, the images shown in Fig. 11, and 5 additional images from $11.5-11.9 \mathrm{~ms}$ are time averaged to further smooth out the image that will be subjected to the inversion. In an ignition case, time averaging can still be performed but there are limitations on the number of images that can be used due to the transient nature of the ignition event. However, a way to increase the number of images used for averaging during ignition is by using a high framing rate in the camera used for data acquisition.

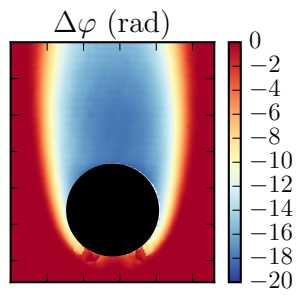

(a) Single frame

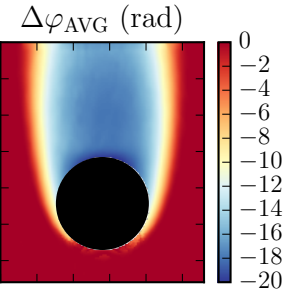

(b) Time averaged

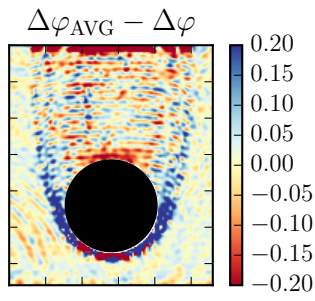

(c) Difference

Figure 12: (a) Single frame optical phase difference taken at $11.8 \mathrm{~ms}$, (b) time averaged optical phase difference, and (c) difference between time averaged and single frame optical phase difference.

A comparison of the optical phase difference of an averaged image against the difference of an averaged image with a single frame image at $11.8 \mathrm{~ms}$ is shown in Fig. 12. It should be noted that Fig. 12 (c) will look the same when com- 
pared against other single frame images. The time averaging served to smooth out fringe artifacts (horizontal faded fringes) that are present after phase unwrapping. It should also be noted that the quality of the optical phase difference goes down in the rear and front stagnation points of the sphere. In the front, the phase demodulation is not able to capture the sharp shifts in the fringes, and in the rear problems of diffraction arise due to astigmatism in the optical system.

\subsection{Abel Transform}

The inverse Abel transform, shown in Eq. 4, is used to obtain the index of refraction fields and subsequently the temperature fields. The inversion of the Abel transform is achieved by using the Nestor-Olsen numerical algorithm [18] that was implemented in the inversion of radially resolved intensity measurements by [19]. The Nestor-Olsen method approximates Eq. 4 by,

$$
f(r)=\frac{-2}{\pi \Delta x} \sum_{i=j}^{N-1} F\left(x_{i}\right) B_{j, i}
$$

where,

$$
B_{j, i}= \begin{cases}A_{j, i-1}-A_{j, i}, & \text { for } i \geq j+1 \\ -A_{j, i}, & \text { for } i=j\end{cases}
$$

and,

$$
A_{j, i}=\frac{\left[i^{2}-(j-1)^{2}\right]^{1 / 2}-\left[(i-1)^{2}-(j-1)^{2}\right]^{1 / 2}}{2 i-1}
$$

where $i$ and $j$ correspond to indices in the $x$ and $y$ directions, respectively.

The refractive index is found after obtaining $f(r)$, using Eqs. 19 and 20, and 
manipulation of Eq. 5, which yields,

$$
n(r)=n_{0}+\frac{\lambda}{2 \pi} f(r)
$$

Subsequently, Eqs. 6 and 7 are used to obtain the temperature fields shown in Fig. 13. All the typical features of this flow become visible after performing the Abel inversion: thermal boundary layer growth from the front stagnation point towards the region of flow separation, shallower temperature gradients where the thermal boundary layer separates (see $y=2 \mathrm{~mm}$ on Fig. 13), and the hot wake left by the passage of the sphere through the gas. Moreover, note the level of detail accomplished in the vicinity of the sphere for $-0.5<y<3 \mathrm{~mm}$ as we are effectively resolving the thermal boundary layer thickness. Some of the difficulties, also evident in this figure (e.g. front and back stagnation points), will be discussed in the next section.

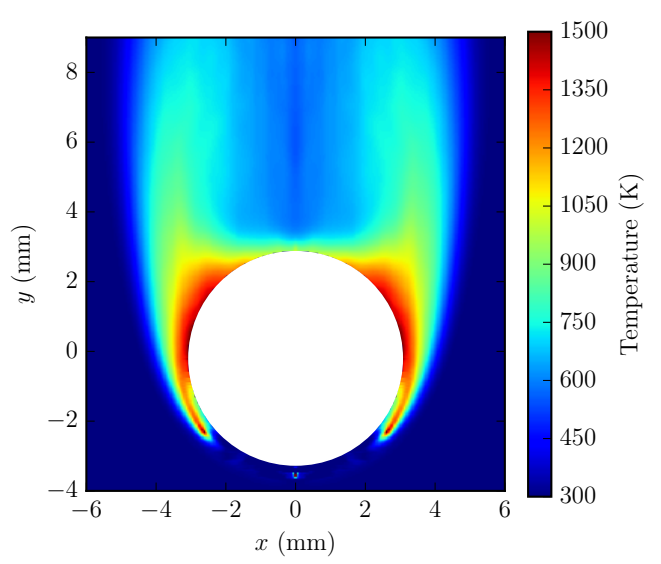

Figure 13: Experimental temperature field. 


\section{Discussion}

Figure 14 shows the temperature field from Fig. 13 compared against numerical simulations of an unconfined $6 \mathrm{~mm}$ diameter sphere with a surface temperature of $1433 \mathrm{~K}$. The numerical methodology is the same as that described in $[7,8,20,21]$ except that two mesh configurations were tested. The first mesh geometry is labeled "unconfined" and corresponds to a large domain such that the wake of the sphere is not affected by wall effects. The second mesh geometry labeled "confined" corresponds to the sphere traveling through a $23 \mathrm{~mm}$ cylinder to reproduce the experimental setup as closely as possible. As indicated in the previous section, the temperature field at the front stagnation point of the sphere cannot to be resolved because the fringes cannot be resolved in that region during the phase demodulation because they are too sharp; meaning, the fringes become vertical corresponding to an infinite shift in phase. Additionally, the rear stagnation point is not resolved because of issues with diffraction that are the result of astigmatism in the optical setup. This section will focus on the region that is well resolved in close proximity to the sphere. More importantly, this is the region were ignition is experimentally [9] observed and numerically [20, 21] predicted to take place when the temperature of the sphere is close to the ignition threshold of a given reactive mixture. Figure 14 shows sections taken from Fig. 13 of the regions surrounding the sphere that are reasonably well resolved qualitatively.

A better way to observe the differences between the numerical and experimental temperature fields is by taking slices along the $y$-axis, this is shown in Fig 15 (a) and (b) for the vicinity and wake of the sphere, respectively.

Figure 15 (a) shows excellent agreement in the vicinity of the sphere up until the rear stagnation point of the sphere, labeled $y=3.0 \mathrm{~mm}$. The shaded horizontal 


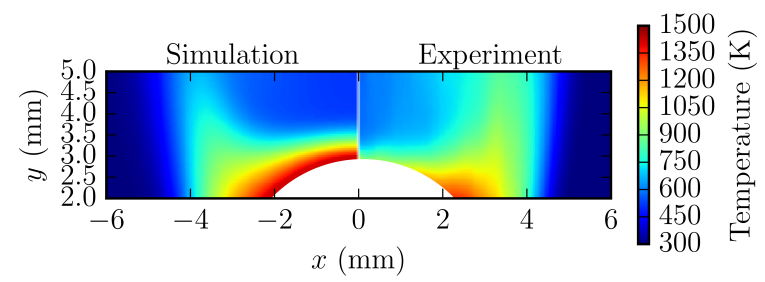

(a) Rear stagnation point

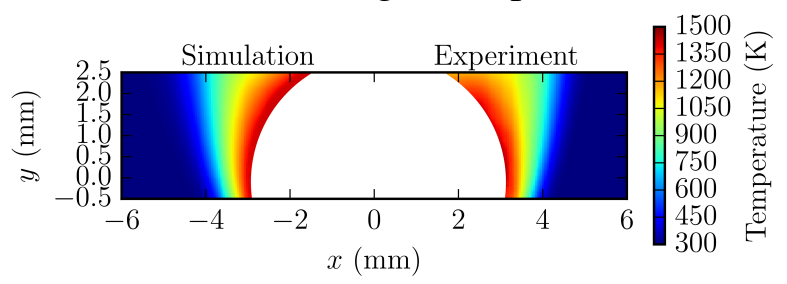

(b) Sphere

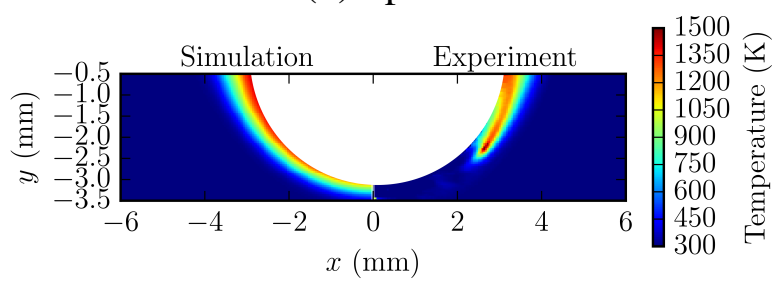

(c) Front stagnation point

Figure 14: Comparison of numerical temperature field (left) with experimental temperature field (right)

lines correspond to upper/lower bounds obtained from pyrometer measurements made of the sphere surface. The lighter shaded regions represent the absolute lower and upper bounds of the numerical temperature fields taken from simulations run using confined and unconfined configurations at the lower and upper bounds of the pyrometer surface temperature of $1308 \mathrm{~K}$ and $1558 \mathrm{~K}$.

Fig. 15 (b) shows slightly higher temperature readings in the experiment than in the simulation. This is to be expected since in the experiment the sphere travels through a confined space (the cylinder) before exiting into an open space. Therefore, the thermal and momentum boundary layers are affected by this confinement 


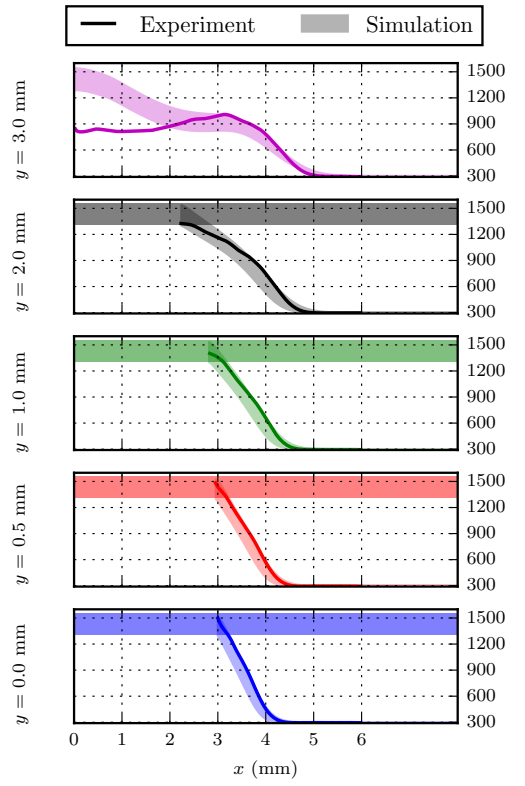

(a) Sphere vicinity

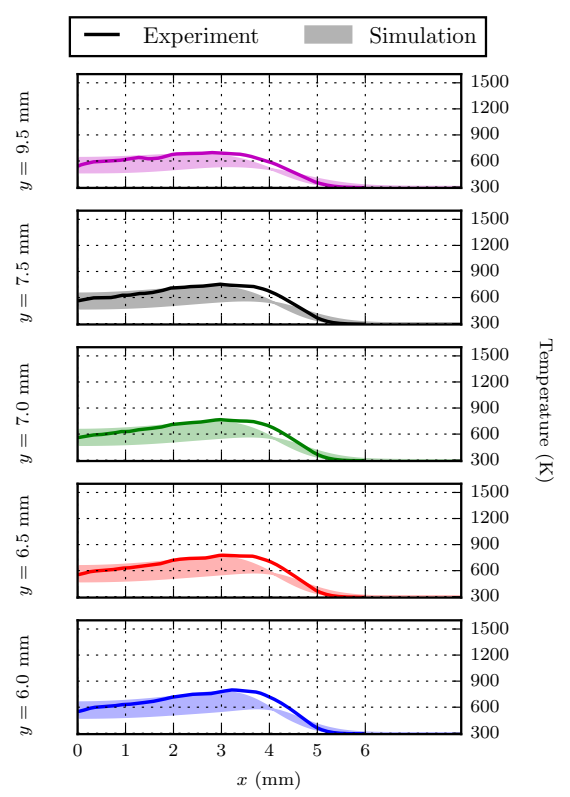

(b) Sphere wake

Figure 15: Slices taken along $y$ axis to compare experimental and numerical temperature fields in the (a) vicinity and (b) wake of the sphere.

resulting in longer wakes when compared to the simulation results. We are yet to determine numerically what the effect of the sphere traveling from confined then to unconfined space is on the temperature fields in the vicinity and wake of the sphere. This could account for the discrepancies that are observed in the wake of the sphere between the numerical and experimental results. Nonetheless, the results from Fig. 15 indicate that the unconfined simulations yield a wider wake and lower temperatures in the wake of the sphere. However, the confined simulations result in a thinner wake and higher temperatures in the wake of the sphere. In the vicinity of the sphere, the confined simulation results show a thinner thermal boundary layer than the unconfined case.

Finally, for completeness, the magnitude of the errors due to the image post- 
processing and refraction assumptions were quantified. Sources of error can be introduced during the phase demodulation and inversion procedures. The assumptions about light refraction are also a source of error. The error introduced through image processing and refraction assumptions is investigated by creating synthetic interferograms with added noise that represent the noise observed in the experimental interferograms. The synthetic interferograms are generated from synthetic temperature distributions that simulate typical temperature profiles found experimentally. The temperature profile tested is the one observed in the thermal boundary layer of the sphere near the region of flow separation. As mentioned earlier, it is this region that is of interest in thermal ignition applications. To generate the synthetic interferograms, a ray tracing algorithm that accounts for the deviation of rays as they travel through a refracting medium is used to compute the synthetic optical phase difference. The synthetic optical phase difference is then wrapped from $-\pi$ to $\pi$, and subsequently the phase is computed and random noise of $10 \%$ is added to generate the synthetic interferogram. The image post-processing is performed on the synthetic interferogram to compute the temperature field, and the error between the synthetic and processed temperature field is calculated. An error of $2 \%$ is observed in the thermal boundary layer. At the sphere surface, a higher error of $15-30 \%$ is obtained. In the freestream, the error is less than $2 \%$. The addition of noise to the synthetic interferogram appears to alter the distribution of the error, however, it does not affect the magnitude. Therefore, it appears that the major contributions to the error come from the phase demodulation procedure and the inversion algorithm. Based on the uncertainty analysis provided in the Supplementary Material, the assumptions about light refraction are negligible. Additionally, the topic of refraction errors in optical interferometry was exten- 
sively treated by Kahl and Mylin [22]. As discussed in that paper, these errors are minimized by using a focusing interferometer, as in the present experimental setup. A study by Hunter and Schreiber [23], concluded that for a well-focused interferometer, as long as $|n-1|<10^{-2}$, the inverted Abel equation provides an acceptably accurate radial refractive index distribution.

Notably, and despite the drawbacks mentioned, interferometry is capable of obtaining accurate results in close proximity to the sphere surface, this is evident by the results shown in Fig. 15 (a).

\section{Conclusions}

The methodology to extract gas temperature field measurements using interferometry was explained in detail and applied to the canonical problem of a hot sphere (6 $\mathrm{mm}$ in diameter) falling through an inert gas. The temperature fields obtained experimentally in close proximity to the sphere, and in the hot wake left by the passage of the sphere through the gas were compared against numerically predicted fields. Good qualitative agreement between experiments and simulations was obtained with all the typical features of this flow being properly revealed by the post-processing methodology described (i.e. thermal boundary layer growth from the front stagnation point towards the region of flow separation, shallower temperature gradients where the thermal boundary layer separates, and hot wake in the zone of flow recirculation). However, poor qualitative agreement was obtained at the front and back stagnation points of the sphere. The high temperature gradients at the front of the sphere result in almost vertical fringes in this region which yield optical phase differences during post-processing that tend to approach infinity. On the other hand, the source of the poor performance of the method at 
the back stagnation point is due to the astigmatism present in the optical setup. A more stringent test was also carried out during this study in which the experimental temperature distributions within the temperature boundary layer and hot wake were quantitatively compared with those determined numerically. Excellent agreement was achieved away from the front/back stagnation points, both in close proximity to the sphere and in the hot wake. The boundary layer thickness along the sphere matched very closely with the numerical simulations; in the wake some minor quantitative differences in temperature are observed but the thickness of the wake was captured perfectly. The overall qualitative and quantitative agreement between experiments and simulations provides positive evidence of the adequacy of the methodology described here, and the capabilities of interferometry to resolve in great detail thermal flows. Magnitude of errors in the thermal boundary layer were found to be on the order of $2 \%$. Future work will seek to alleviate the issues encountered with the post-processing by making improvements to the optical setup to achieve an astigmatism-free optical system to better resolve the back stagnation point. Additionally, a different numerical simulation comparison will be made by simulating a sphere going from a confined to unconfined space, similar to what is observed in the experimental setup. Finally, the application of this methodology to reactive cases and a frame work for error estimation for temperature fields obtained with interferometry will be the topic of an upcoming manuscript.

\section{Acknowledgments}

This work was carried out in the Explosion Dynamics Laboratory of the California Institute of Technology, and was supported by The Boeing Company through 
a Strategic Research and Development Relationship Agreement CT-BA-GTA-1.

[1] P. Hariharan, Basics of Interferometry, Academic Press, Burlington, second edition, 2007.

[2] D. L. Reuss, Combustion and Flame 49 (1983) 207 - 219.

[3] X. Xiao, C. W. Choi, I. K. Puri, Combustion and Flame 120 (2000) 318 332.

[4] M. Irandoost, M. Ashjaee, M. Askari, S. Ahmadi, Optics and Lasers in Engineering 74 (2015) $94-102$.

[5] Z. N. Ashrafi, M. Ashjaee, M. Askari, Optics Communications 341 (2015) $55-63$.

[6] C. Qi, S. Zheng, H. Zhou, International Journal of Thermal Sciences 115 (2017) $104-111$.

[7] J. Melguizo-Gavilanes, A. Nové-Josserand, S. Coronel, R. Mével, J. Shepherd, Combustion Science and Technology (2016).

[8] J. Melguizo-Gavilanes, L. Boeck, R. Mével, J. Shepherd, International Journal of Hydrogen Energy (2016).

[9] S. A. Coronel, Thermal Ignition Using Moving Hot Particles, Ph.D. thesis, California Institute of Technology, 2016.

[10] W. Z. Black, W. W. Carr, Review of Scientific Instruments 42 (1971) $337-$ 340.

[11] W. Merzkirch, Flow Visualization, Academic Press, 1987. 
[12] A. D. Poularikas (Ed.), Tranforms and Applications Handbook, CRC, 2010.

[13] W. Gardiner, Y. Hidaka, T. Tanzawa, Combustion and Flame 40 (1981) 213 $-219$.

[14] P. Rastogi, E. Hack (Eds.), Phase Estimation in Optical Interferometry, CRC Press, 2015.

[15] Q. Kemao, Windowed Fringe Pattern Analysis, SPIE, 2013.

[16] T. A. Johnson, V. C. Patel, Journal of Fluid Mechanics 378 (1999) 19-70.

[17] D. Ghiglia, M. D. Pritt, Two-Dimensional Phase Unwrapping: Theory, Algorithms, and Software, John Wiley and Sons, Inc., 1988.

[18] O. H. Nestor, H. N. Olsen, SIAM Review 2 (1960) 200-207.

[19] R. Álvarez, A. Rodero, M. C. Quintero, Spectrochimica Acta Part B: Atomic Spectroscopy 57 (2002) 1665-680.

[20] J. Melguizo-Gavilanes, S. Coronel, R. Mével, J. Shepherd, International Journal of Hydrogen Energy (2016).

[21] J. Melguizo-Gavilanes, R. Mével, S. Coronel, J. Shepherd, Proceedings of the Combustion Institute 36 (2017) 1155 - 1163.

[22] G. Kahl, D. C. Mylin, Journal of the Optical Society of America 55 (1965) 364-\&.

[23] A. M. Hunter, P. W. Schreiber, Appl. Opt. 14 (1975) 634-639. 